\title{
Checks and balances in neuromodulation
}

\section{Ronald M. Harris-Warrick* and Bruce R. Johnson}

Department of Neurobiology and Behavior, Cornell University, Ithaca, NY, USA

Edited by:

Kathleen A. French, University of

California San Diego, USA

Reviewed by:

David Schulz, University of Missouri-

Columbia, USA

\section{*Correspondence:}

Ronald M. Harris-Warrick, Department

of Neurobiology and Behavior

Seeley G. Mudd Hall, Cornell

University, Ithaca, NY 14853, USA.

e-mail:rmh4@cornell.edu

\begin{abstract}
Neuromodulators such as monoamines and peptides play important roles in activating and reconfiguring neural networks to allow behavioral flexibility. While the net effects of a neuromodulator change the network in a particular direction, careful studies of modulatory effects reveal multiple cases where a neuromodulator will activate functionally opposing mechanisms on a single neuron or synapse. This review gives examples of such opposing actions, focusing on the lobster pyloric network, and discusses their possible functional significance. One important action of opposing modulatory actions may be to stabilize the modulated state of the network, and to prevent it from being overmodulated and becoming non-functional.
\end{abstract}

Keywords: neuromodulation, ion channel, synapse, central pattern generator, network, opposition
Opposing cellular and synaptic mechanisms are universal in the nervous system. Inhibition plays an equally important role with excitation in shaping brain function and neural network output; for instance, pharmacological blockade of inhibitory synaptic activity leads to uncontrolled seizures. At the cellular level, the membrane potential is continually shaped by opposing ionic currents; for example, the shape of the depolarizing trajectory of pacemaker neurons is determined by the relative amplitudes of opposing subthreshold currents, such as the transient potassium current $\left(I_{\mathrm{A}}\right)$ and the hyperpolarization-activated inward current, $I_{\mathrm{h}}$ (Harris-Warrick et al., 1995b; see also Balu and Strowbridge, 2007). At the circuit level, the intricate interaction between excitatory and inhibitory synaptic inputs shapes alternating flexor and extensor activity in vertebrate spinal locomotor networks (Endo and Kiehn, 2008). Over the long term, opposing homeostatic mechanisms such as synaptic scaling assure stability in synaptic strength (Turrigiano, 2008) as well as the firing properties of neurons (MacLean et al., 2003; Schulz et al., 2007).

In this review, we discuss the possible functions of opposing actions of neuromodulators on neural networks. Neuromodulators are transmitters that activate metabotropic (typically G-protein coupled) receptors to activate second messenger cascades that fundamentally alter the biochemistry of the target neuron. As a consequence, the activity of multiple proteins, including ion channels, receptors and enzymes, is simultaneously altered, reconfiguring the firing properties of the neuron as well as its synaptic interactions with other neurons. This reconfigures the output of neural networks, facilitating the behavioral flexibility which is essential for an animal to survive. Few researchers have systematically attempted to identify all the molecular targets of a neuromodulator in a neuron or a network; most studies have attempted to identify the changes that are consistent with the overall physiological consequences of modulator action. However, in an increasing number of cases, opposing neuromodulatory actions have been identified, where some of the changes evoked by a neuromodulator support the net physiological effect while others actively oppose it. Here we discuss a number of examples of opposing actions of a single neuromodulator at the cellular, synaptic, and network level of organization, to try to understand why such opposing actions exist.
Most of our examples come from our detailed studies of the cellular and biophysical mechanisms by which three monoamines, dopamine (DA), serotonin, and octopamine, reconfigure the pyloric network in the stomatogastric ganglion in the lobster Panulirus interruptus (Harris-Warrick et al., 1998; Harris-Warrick and Johnson, 2002). This is an ideal model system for studying the multiple mechanisms of neuromodulation (Harris-Warrick et al., 1992; Johnson and Hooper, 1992). The pyloric network drives rhythmic movements of the crustacean foregut. It contains only 14 neurons, each of which can be identified, isolated from all synaptic input and studied as an individual. All the synaptic connections between these neurons have been mapped and can be individually studied (Figure 1B). The pyloric network generates a simple rhythmic motor pattern that can be recorded in vitro by the isolated stomatogastric nervous system (Figure 1A). DA, serotonin, and octopamine, as well as several peptides and other modulators, can elicit a unique motor pattern from the quiescent pyloric network in the isolated STG (Flamm and Harris-Warrick, 1986a; Marder and Bucher, 2007), and can elicit unique and reproducible changes in the ongoing network with descending modulatory inputs intact (Figure 1A). DA, serotonin, and octopamine each directly modulate nearly all of the pyloric neurons, each with a variety of different effects on different neurons, ranging from simple inhibition to evoking rhythmic bursting (Flamm and Harris-Warrick, 1986b). Similarly, each amine increases or decreases the strengths of nearly all the synapses in the network, effectively "rewiring" it for a new behavior (Johnson et al., 1995). Detailed voltage clamp and calcium imaging studies have revealed a remarkably complex set of modulatory effects of each amine (Figure 1C). Among these effects are many examples of a neuromodulator having opposing actions on a single cell or synapse (Figures 1C and 5). In this review, we describe examples of these opposing modulatory actions at the cellular, the synaptic, and the network level of organization. We also include examples from other systems where similar opposition has been described.

\section{OPPOSITION AT THE CELLULAR LEVEL}

In the pyloric rhythm, the ventricular dilator (VD) interneuron fires rhythmic bursts of action potentials that are shaped by synaptic inhibition from the anterior burster $(\mathrm{AB})$, pyloric dilator 


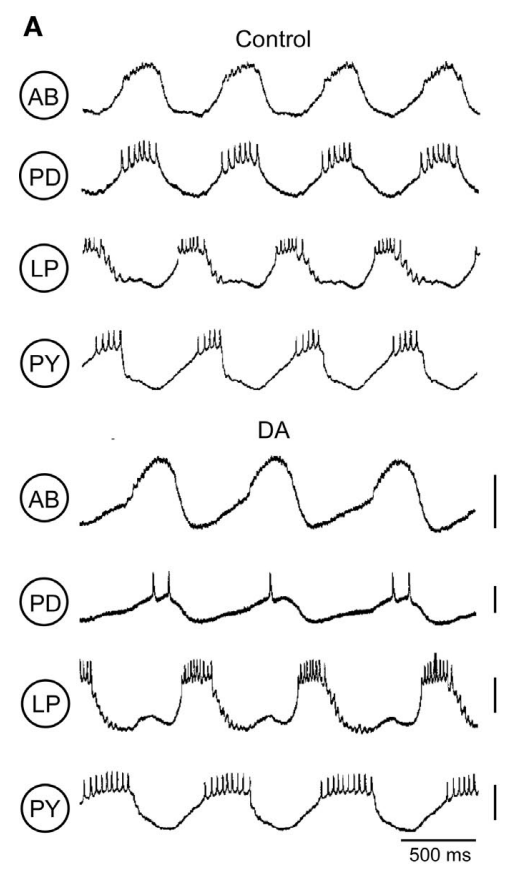

FIGURE 1 | Dopamine (DA, $10^{-4} \mathrm{M}$ ) modulation of pyloric network activity. (A) Comparison of pyloric activity in $A B, P D, L P$, and PY neurons with descending modulatory inputs intact before (Control) and during DA application (DA). Voltage markers, $10 \mathrm{mV}$ (modified from Kloppenburg et al., 2008). (B) Pyloric network of the spiny lobster Panulirus interruptus showing the synaptic connections between the major classes of pyloric network neurons. Synaptic connections

B

C
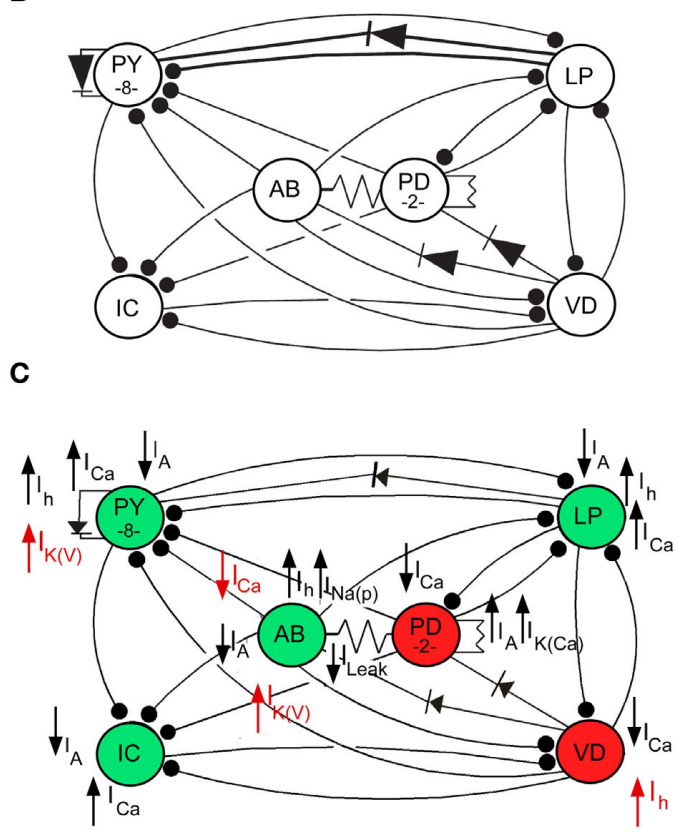

are electrical (non-rectifying, resistor symbols; rectifying, diode symbols) and chemical inhibitory (filled circles). (C) Known ionic current targets of DA in the pyloric neurons. Currents are strengthened (upward arrows) or weakened (downward arrows) by DA. Green and red neurons indicate enhanced and reduced excitability with DA. Currents in red act in opposition to the net effects of DA on the excitability of the different neurons.
(PD), and lateral pyloric (LP) neurons. When isolated from all synaptic input, it fires tonically at low frequencies. Bath application of DA typically hyperpolarizes and silences the VD neuron (Flamm and Harris-Warrick, 1986a,b; Figure 2A). This inhibition is mediated in part by a reduction in the voltage-dependent calcium current, $I_{\mathrm{Ca}(\mathrm{V})}$ (Johnson et al., 2003), and in many VD neurons, enhancement of the 4-aminopyridine-sensitive transient potassium current, $I_{\mathrm{A}}$ (Harris-Warrick et al., 1998). Despite this overall inhibitory effect, voltage clamp studies showed that DA enhances the hyperpolarization-activated inward current, $I_{\mathrm{h}}$, shifting its voltage of half activation by $8 \mathrm{mV}$ in the depolarizing direction (Peck et al., 2006; Figure 2B). This increases the $I_{\mathrm{h}}$ active in the physiologically relevant voltage range, which would act to depolarize the neuron, opposing the predominantly inhibitory effect of DA. Further study showed that the kinetics of these opposing effects may be different. In a majority of synaptically isolated VD neurons, a brief puff of DA evoked a brief reduction followed by a prolonged enhancement in firing rate (Figure $2 \mathrm{C}$ ). The brief reduction was blocked by the $I_{\mathrm{A}}$ blocker 4-aminopyridine, while the prolonged enhancement was blocked by the $I_{\mathrm{h}}$ blocker $\mathrm{CsCl}$. Thus, these apparently opposing effects may shape a complex biphasic temporal response to brief activation of dopaminergic neurons providing input to the STG.

A similar example of opposing effects with different kinetics was reported by Power and Sah (2008). In neurons in the rat basolateral amygdala, spike frequency and bursting are regulated by a slow afterhyperpolarization mediated by two different calcium-activated potassium currents, $I_{\mathrm{SK}}$ and $s I_{\mathrm{AHP}}$. Cholinergic inputs dynamically regulate the AHP shape, and thus the spike frequency, by two opposing mechanisms: a muscarinic suppression of the $s I_{\mathrm{AHP}}$ and a muscarinic enhancement of $I_{\mathrm{SK}}$ mediated by an $\mathrm{IP}_{3}$ pathway. The inhibitory effect predominates during bath application of ACh but the excitatory effect predominates during short focal application onto the soma and proximal dendrites. Thus the sign of the ACh effect depends on the location and duration of the cholinergic input to these neurons.

Another example from the pyloric network uses opposing modulatory actions to regulate spike frequency during bursting. DA excites the majority of the pyloric constrictor (PY) neurons as well as the $A B$ neuron. These neurons increase their spike frequency, due in part to reductions in $I_{\mathrm{A}}$ (Harris-Warrick et al., 1995a; Peck et al., 2001). However, DA also enhances a high-threshold voltage-activated potassium current, $I_{\mathrm{K}(\mathrm{V})}$ in these neurons (Gruhn et al., 2005; Figure 1C). This current is only activated during action potentials and, due to its slow deactivation rate, will function to limit the maximal spike frequency of the DA-excited neurons by prolonging their absolute refractory period. The opposing effects of DA to reduce $I_{\mathrm{A}}$ but enhance $I_{\mathrm{K}(\mathrm{V})}$ result in a constrained increase in spike frequency that will not become too high to disrupt network function. Thus, these opposing actions of DA protect the network from a positive feedback loop of excitation.

The $\mathrm{AB}$ neuron shows very complex responses to DA. This neuron is a conditional oscillator that serves as the primary pacemaker to drive the pyloric rhythm. AB bursting is carefully regulated by 


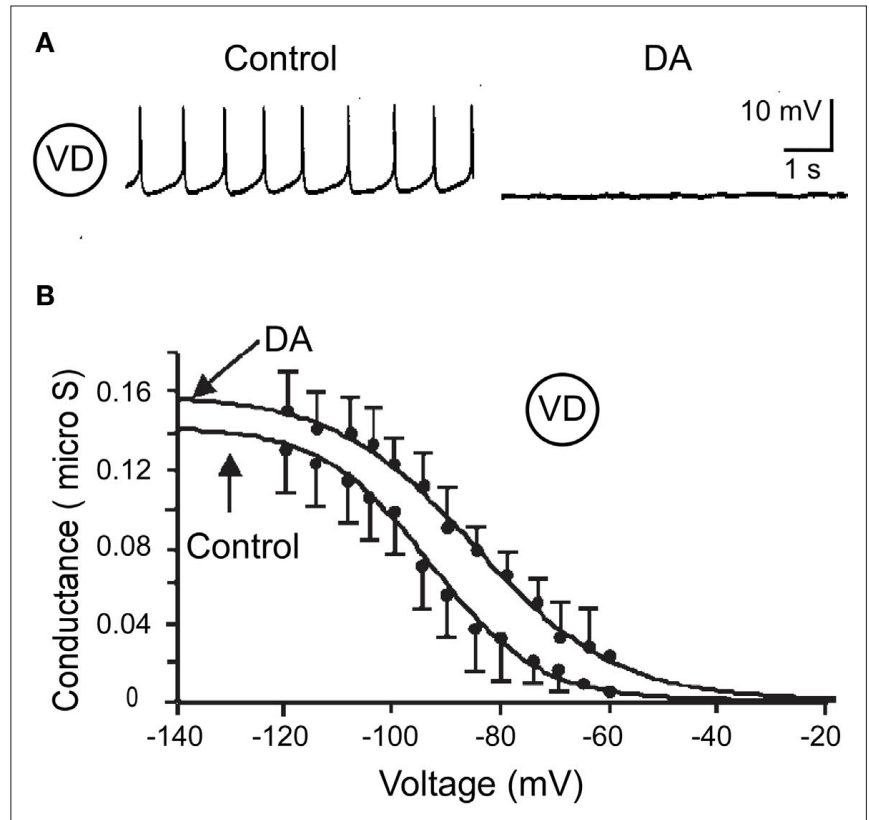

C

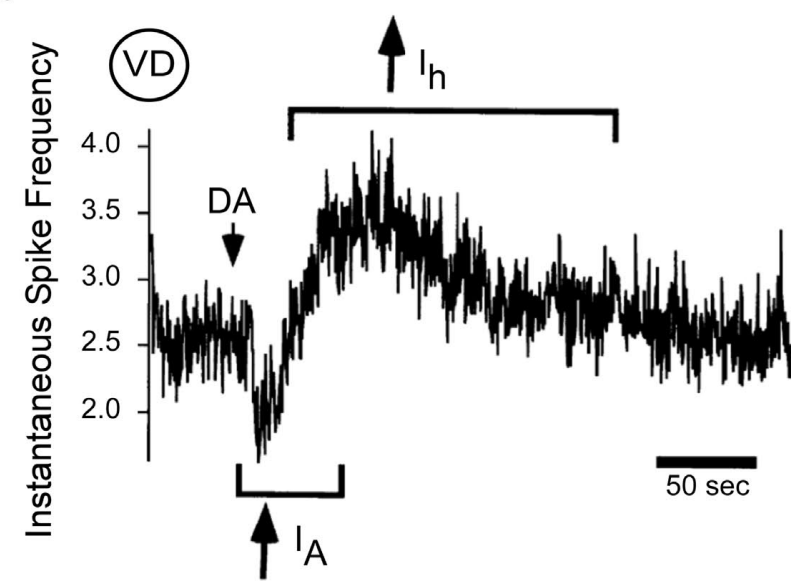

FIGURE 2 | Dopamine $\left(10^{-4} \mathrm{M}\right)$ modulates VD neuron excitability.

(A) Direct DA inhibition of a synaptically isolated VD neuron (modified from Flamm and Harris-Warrick, 1986b). (B) Effects of DA on $I_{n}$ activation in the VD neuron (modified from Peck et al., 2006). (C) Biphasic effect of DA on VD spike frequency; the initial spike frequency reduction is blocked by $4 \mathrm{mM} 4-\mathrm{AP}\left(\mathrm{I}_{\mathrm{A}}\right)$ and the delayed enhancement of spike frequency is blocked by $5 \mathrm{mM} \mathrm{CsCl}\left(I_{h}\right)$ (modified from Harris-Warrick et al., 1998).

multiple neuromodulators, which modify different combinations of ionic currents to evoke different oscillation rates and amplitudes, and drive the motor pattern at different speeds. DA-induced bursting requires an increase in intracellular calcium (HarrisWarrick and Flamm, 1987; L. Kadiri and R. Harris-Warrick, in preparation), which is released by DA from intracellular stores before the neuron starts to oscillate. DA-induced bursting is abolished by the calcium chelator BAPTA, or by drugs that block calcium release from intracellular stores. Although calcium entry via plasma membrane $I_{\mathrm{Ca}(\mathrm{V})}$ channels would be expected to contribute to this increase in intracellular calcium, we found that DA instead dramatically reduces $I_{\mathrm{Ca}(\mathrm{V})}$ by over $50 \%$ (Johnson et al.,
2003). This action could serve to protect the neuron from too great an elevation in intracellular calcium, which would activate $I_{\mathrm{K}(\mathrm{Ca})}$ and terminate bursting.

Levitan and colleagues (Levitan et al., 1987; Levitan and Levitan, 1988) described another regulation of bursting activity in Aplysia: both the peptide egg-laying hormone (ELH) and serotonin activate opposing currents in the burster neuron, R15. Lower 5-HT concentrations enhance an inward-rectifying potassium current, $I_{\mathrm{KIR}}$, which prolongs the hyperpolarizing phase of R15 bursts, slowing the burst frequency. Higher 5-HT concentrations additionally activate a subthreshold $I_{\mathrm{Ca}(\mathrm{V})}$, which eventually outweighs the potassium current and drives tonic firing. The net effect is to convert R15 from a bursting neuron to a bistable neuron with an up-state of tonic firing, sustained by the elevated $I_{\mathrm{Cav}}$, that can be reversibly switched by weak inhibitory input to the hyperpolarized silent down-state, sustained by the elevated $I_{\text {KIR }}$. Thus, these two opposing currents, which are active in different voltage ranges and show different 5-HT concentration dependence, shape the intrinsic firing properties and responses to synaptic inputs in R15.

\section{OPPOSITION AT THE SYNAPTIC LEVEL}

A single neuromodulator can also evoke changes of opposite sign to regulate the strength of synaptic interactions within a neural network. Over many years of research, we have determined the effects of DA and 5-HT on all the synapses in the pyloric network, studying both pre-synaptic mechanisms that regulate transmitter release and post-synaptic mechanisms that regulate responsiveness to the transmitter. At a number of pyloric synapses, these effects are of opposite sign.

The major synaptic feedback to the pyloric pacemaker neurons is mediated by a glutamatergic graded inhibitory synapse from the LP neuron to the PD neuron (Figure 1B). This LP $\rightarrow$ PD synapse is significantly strengthened by DA (Johnson et al., 1995). When the voltage-clamped pre-synaptic LP neuron is driven by trains of voltage oscillations averaged from its normal bursting behavior, DA enhances both the initial IPSP amplitude and the steady state IPSP amplitude achieved by the end of the train (Figure 3A; B. R. Johnson and R. M. Harris-Warrick, submitted).

Using calcium imaging with multiphoton microscopy, we analyzed DA's effects on voltage-dependent calcium entry into the LP pre-synaptic terminals (Kloppenburg et al., 2007). DA evoked a significant increase in voltage-dependent calcium accumulation at most LP varicosities (Figure 3B). We confirmed this imaging result using voltage clamp to show that DA enhances $I_{\mathrm{Ca}(\mathrm{V})}$ in the LP neuron (Johnson et al., 2003). These results suggest that at least part of DA's enhancement of the LP synapse is pre-synaptic.

To study the post-synaptic effects of DA on the PD neuron, we replaced the LP synapse with iontophoretic application of its transmitter, glutamate, onto a synaptically isolated PD neuron. Even though DA enhanced the LP $\rightarrow$ PD synapse, it dramatically reduced the PD response to iontophoresis of the LP's transmitter, glutamate (Johnson and Harris-Warrick, 1997; Figure 3C). This effect results from a direct reduction in the synaptic response (Cleland and Selverston, 1997) and a general reduction in the input resistance of the PD neuron, which reduces its responsiveness to all synaptic inputs (Johnson and Harris-Warrick, 1997). 


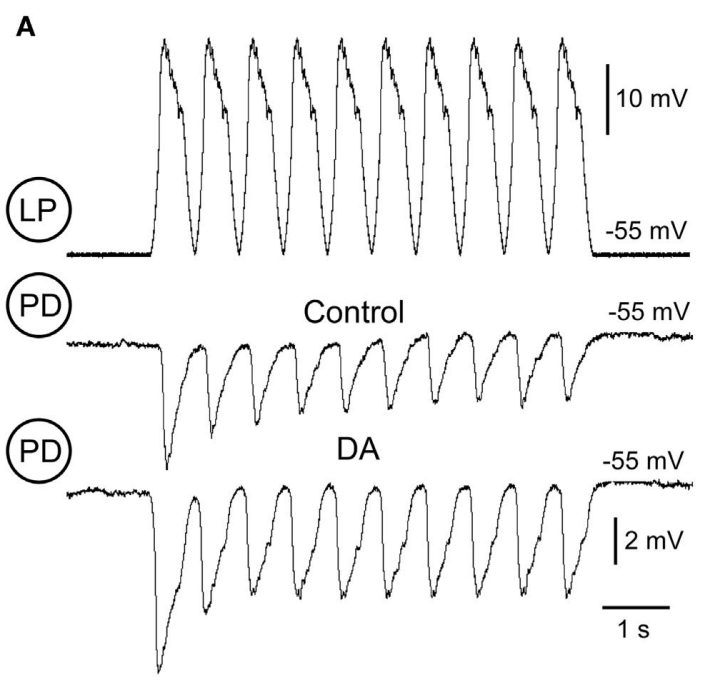

C

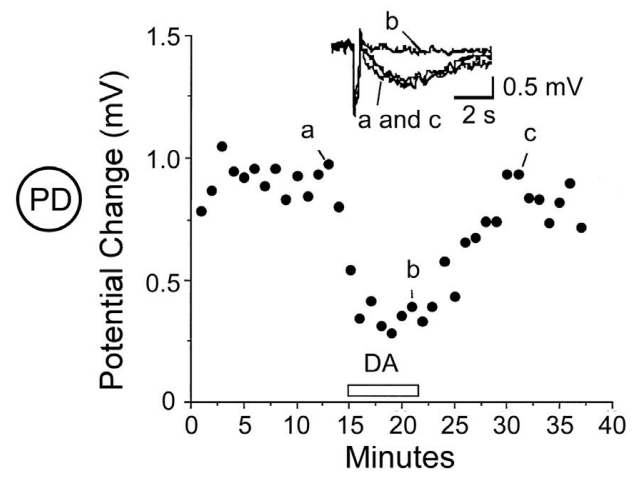

FIGURE 3 | Dopamine modulation of LP $\rightarrow$ PD synaptic transmission.

(A). Pre-synaptic LP depolarization with realistic waveforms (top) and PD responses to this waveform under control conditions (middle) and during application of DA (bottom). (B) Dopamine-induced increase of voltage-activated $\mathrm{Ca}^{2+}$ accumulation in an LP neuron (modified from Kloppenburg et al., 2007). B1 LP neuron loaded with Calcium Green-1, indicating where the line scans were performed (white line) and the varicosity where the change in fluorescence was analyzed (red line). Scale: 10 microns. B2: time course of the DA-induced change of the peak $\mathrm{Ca}^{2+}$ accumulation from the varicosity in $\mathrm{B} 1$, calculated as the percent

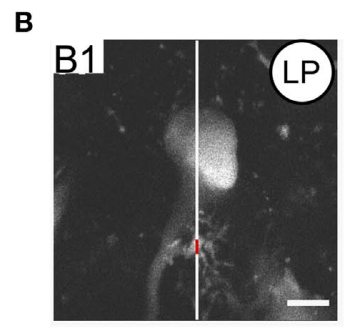

\section{B3}

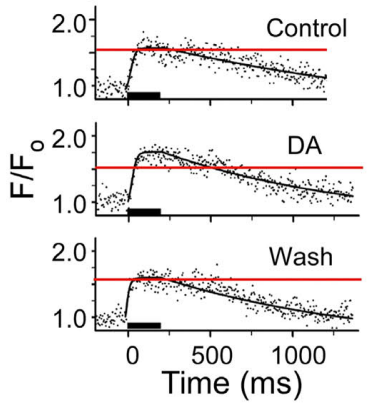

D

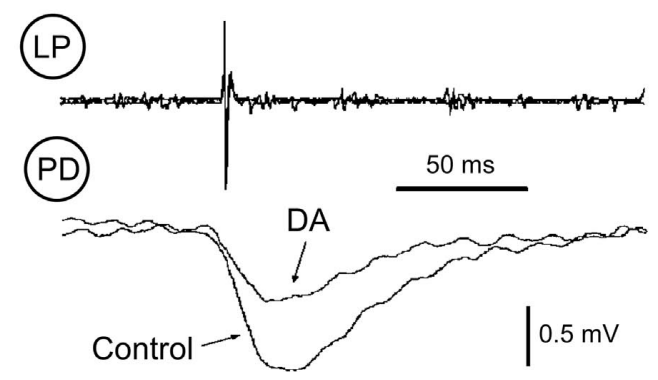

increase in fluorescence over baseline level. B3: Line scans before, during, and after bath application of DA. $\mathrm{Ca}^{2+}$ accumulation was induced by a 200-ms voltage pulse from -45 to $0 \mathrm{mV}$ (black bars). B4: quantified changes in fluorescence extracted from the line scans in B3. (C) DA reduces the PD response to iontophoresis of the LP's transmitter glutamate (Glu). Peak voltage response to Glu is measured every $60 \mathrm{~s}$ before DA (a), during DA (b; bar above the $x$-axis), and during a wash period (c; modified from Johnson and Harris-Warrick, 1997). (D) DA reduces the LP IPSP evoked by an LP action potential, recorded extracellularly on the lateral ventricular nerve (top trace) (modified from Ayali et al., 1998).
Thus, DA enhances the strength of the LP $\rightarrow$ PD synapse, and enhances pre-synaptic calcium currents in LP terminals, but at the same time it dramatically reduces the PD post-synaptic response to LP's transmitter. These opposing actions would function to limit the change in strength of the synapse, and could also stabilize it at its new strength.

There is another novel form of opposition by DA at the LP $\rightarrow$ PD synapse. The pyloric neurons release transmitter by both spikeevoked and graded release, and DA has opposite effects on these two forms of release at the LP output synapses (Ayali et al., 1998). In contrast to the DA enhancement of graded transmission at the $\mathrm{LP} \rightarrow \mathrm{PD}$ synapse described above (Figure 3A), DA reduced spikeevoked transmission at the same time (Figure 3D). This reduction in spike-evoked transmission correlated linearly with DA's postsynaptic reduction of the PD neuron's input resistance. A similar result was observed at the LP $\rightarrow$ VD synapse. These surprising results suggest that there are basic differences between spike-evoked and graded transmitter release at the pyloric synapses. One possibility is that they are mediated by different calcium currents, as has been described in the leech (Lu et al., 1997), and DA modulates one class differently from the other. This mechanism would also stabilize the strength of LP inhibition of the PD neuron, preventing it from disrupting the pyloric rhythm.

The output synapses of the PY neurons illustrate a different form of modulatory opposition. There are eight PY neurons; all their output synapses are significantly strengthened by DA. At PY $\rightarrow$ LP synapses, in the absence of neuromodulators, the synapse often falls silent (Figure 4A). Addition of DA reactivates the synapse so that PY depolarization generates a significant IPSP in the LP neuron (Johnson et al., 1994; Figure 4A). This dopaminergic synaptic 


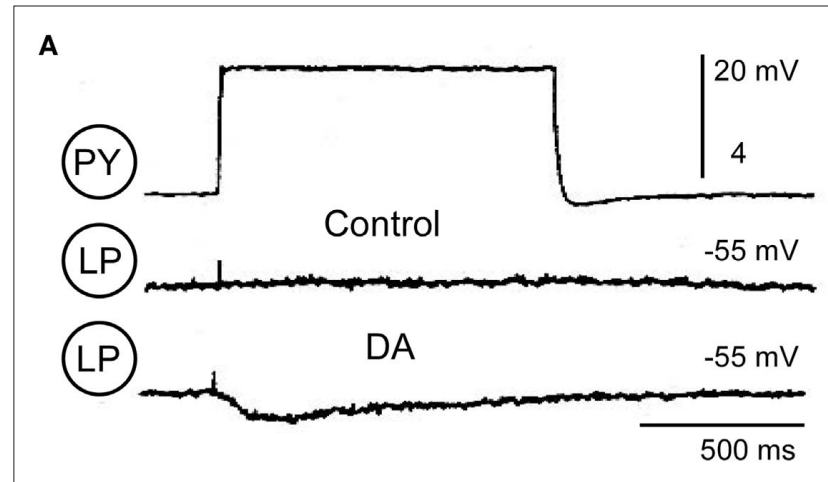

B

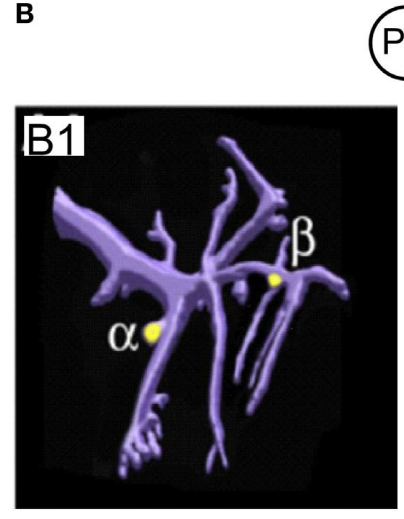

(PY)

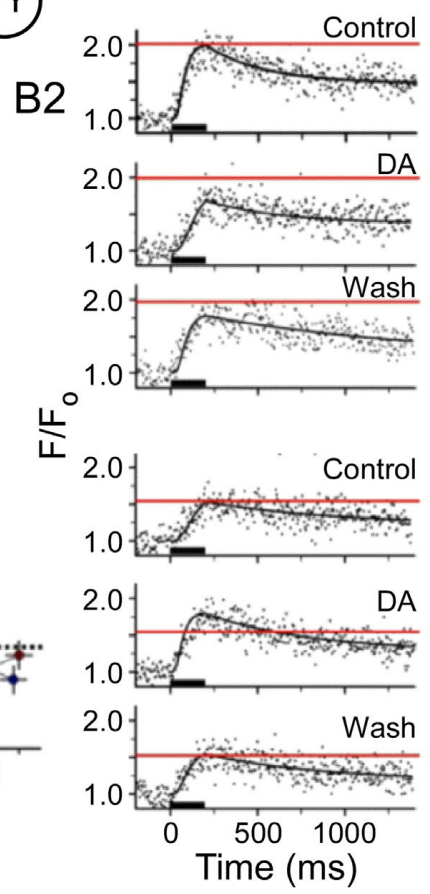

B3

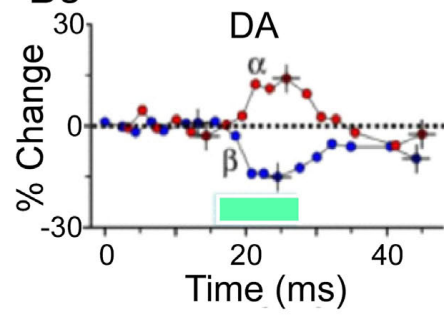

FIGURE 4 | Dopamine (DA, $10^{-4} \mathrm{M}$ ) modulates the PY $\rightarrow$ LP graded synapse. (A) Pre-synaptic PY depolarization (top) and LP responses under control conditions (middle) and during DA (bottom) (modified from Johnson et al., 1994). (B) DA evokes opposing changes in voltage-dependent $\mathrm{Ca}^{2+}$ accumulation in two varicosities on a neurite from a PY neuron (modified from Kloppenburg et al., 2007). B1: Computer reconstruction of an imaged PY neurite, indicating the two varicosities analyzed for $\mathrm{Ca}^{2+}$ entry in response to a 200-ms depolarizing voltage pulse. B2: Quantified changes in percent fluorescence extracted from the line scans at alpha and beta varicosities. Dopamine reduces voltage-activated $\mathrm{Ca}^{2+}$ activation at site alpha and increases the $\mathrm{Ca}^{2+}$ signal at site beta. B3:Time course of the DA-induced changes of the peak $\mathrm{Ca}^{2+}$ accumulation at the two varicosities.

enhancement could occur by pre- and/or post-synaptic mechanisms. Post-synaptically, the isolated LP neuron shows an enhanced response to iontophoresis of the PY transmitter, glutamate, consistent with the strengthened synapse (Johnson and Harris-Warrick, 1997). Pre-synaptically, however, our calcium imaging studies of PY varicosities revealed an unexpected complexity (Kloppenburg et al., 2007). About $40 \%$ of pre-synaptic PY varicosities responded to DA with an enhanced voltage-activated calcium entry, consistent with the strengthened synapse. Another $40 \%$ showed no change at all. However $20 \%$ of the varicosities responded with an unexpected reduction in calcium entry during DA. The eight PY neurons are somewhat heterogeneous, and we initially proposed that these varying responses of PY varicosities to DA arose from the differential DA responses of the PY neuron subtypes. However, this turned out to be wrong, because opposing actions of DA could be seen on different varicosities from a single PY neuron. In 17 experiments we simultaneously imaged calcium accumulation in two varicosities of a single PY neuron; we found all possible combinations of DA effects on calcium accumulation in the two sites. In six neurons, the calcium response increased with DA at one varicosity but decreased at the other varicosity (Figure 4B).

The PY synapses thus provide a different potential mechanism for opposing effects of DA to regulate synaptic strength. Only a subset of the PY terminals show increased voltage-dependent calcium accumulation, while a significant number of varicosities showed no change or the opposite, a decrease in calcium accumulation. On average, the net response is an increase in calcium entry, which was confirmed in somatic voltage clamp measurements of $I_{\mathrm{Ca}(\mathrm{V})}$ (Johnson et al., 2003). One possible interpretation of these data is that DA is regulating the strength of the PY output synapses, not by having opposing pre- and post-synaptic effects, but by having opposing pre-synaptic effects in different PY terminals. In $P$. interruptus, each pyloric neuron makes multiple physical synaptic contacts onto each of its post-synaptic target neurons (King, 1976; P. Kloppenburg, unpublished). If some of these terminals release more transmitter while others release less during DA application, the net effect will be to limit the maximal increase in synaptic strength. Again, this may function to limit the degree of modulation of these synapses and retain the network in a functional state.

Such opposing changes in DA modulation of synaptic strength are not rare in the pyloric network. As seen in Figure 5, the red circled synapses are those where we have detected opposing effects of DA. Opposing effects of a neuromodulator on synaptic strength are occasionally seen in other systems as well. For example, Goldfarb et al. (1993) showed that serotonin, acting on different receptors, simultaneously enhances and inhibits glutamate's ability to evoke release of norepinephrine in the hypothalamus.

\section{OPPOSITION AT THE NETWORK LEVEL}

Opposition can also occur at multiple levels of organization in a neural network, driving unexpected changes in network function. These changes arise from complex interactions between the modulator's effects on the intrinsic firing properties of the neurons and on the strengths of synapses in the network. Because this is complicated and system-specific, we provide just two examples to illustrate the phenomenon.

As shown in Figure 3, DA enhances the overall strength of the $\mathrm{LP} \rightarrow$ PD synapse by complex and opposing pre- and post-synaptic effects. Since the LP neuron provides the only chemical synaptic feedback to the AB-PD pacemaker kernel, DA's enhancement of the $\mathrm{LP} \rightarrow \mathrm{PD}$ synapse would be expected to enhance LP regulation of the cycle period. This was tested by measuring the cycle frequency before and during hyperpolarization of the LP neuron, to eliminate its synaptic inhibition of the PD neurons (B. Johnson and R. Harris-Warrick, submitted). Surprisingly, instead of strengthening 


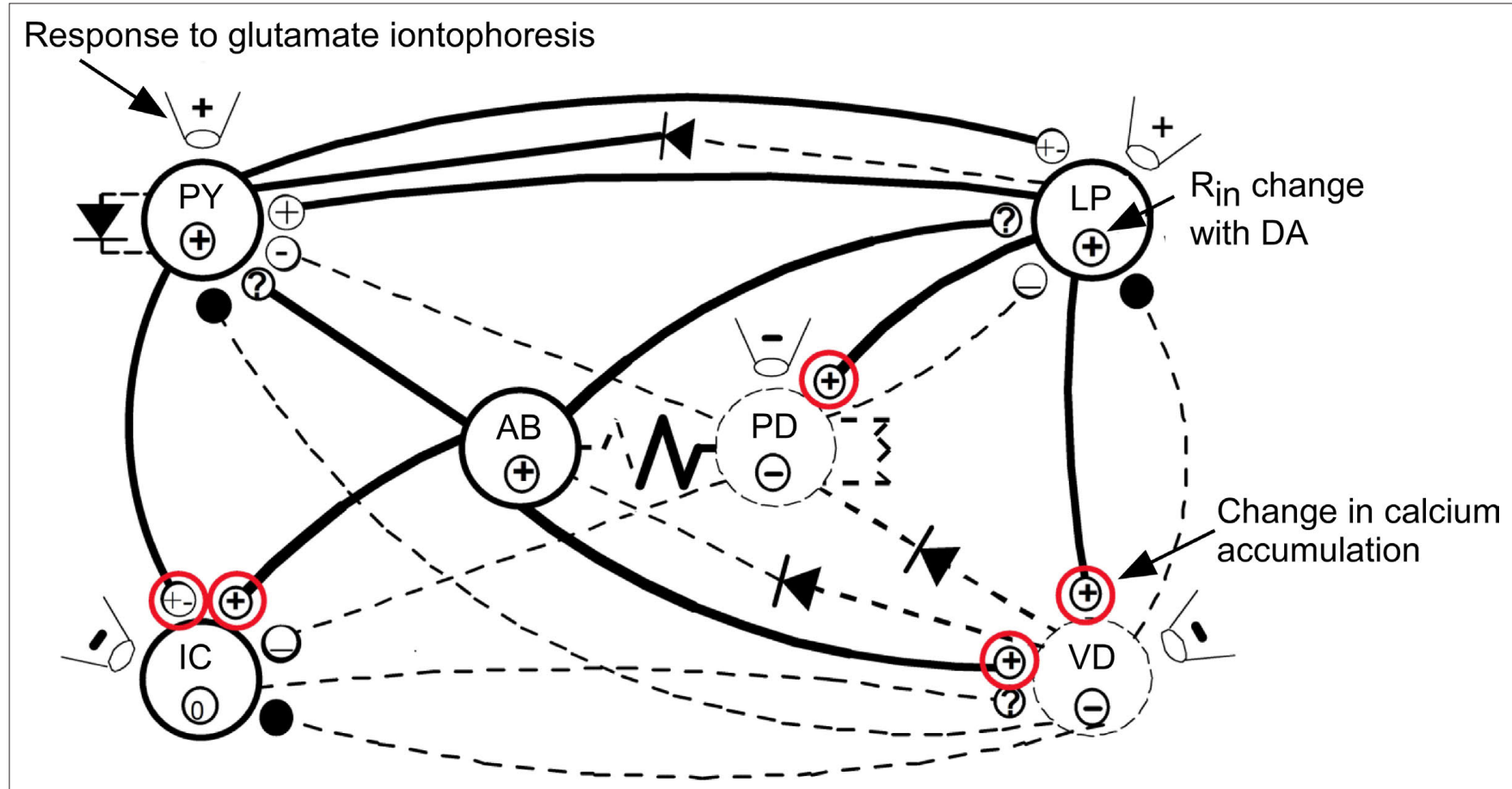

FIGURE 5 | Summary of the effects of DA on pyloric network connections (modified from Harris-Warrick et al., 1998). Synapses strengthened or weakened by DA are shown with bold or dashed lines. Effects of DA on input resistance shown by circled symbols within each neuron. DA effects on neuronal responses to glutamate iontophoresis shown in the pipette symbols next to each neuron. Known DA effects on transmitter release and $\mathrm{Ca}^{2+}$ accumulation are shown in the terminal symbol for synapses (modified from Harris-Warrick et al., 1998). Red circles indicate synapses with opposing pre- and post-synaptic actions of DA. its control of cycle frequency during DA, the LP $\rightarrow$ PD synapse loses its ability to slow the cycle period. This arises from a phase shift in the timing of LP inhibition. The AB/PD oscillators can be phase-advanced or phase-delayed by appropriately timed synaptic inhibition, which can thus change the cycle frequency (Ayali and Harris-Warrick, 1999; Prinz et al., 2003; Thurumalai et al., 2006). Phase-response curves reflect this, with a null point at a particular phase in the cycle when inhibition has no effect. DA excites the LP by a reduction of $I_{\mathrm{A}}$ and an enhancement of $I_{\mathrm{h}}$ (Figure 1C). These currents accelerate the rate of LP post-inhibitory rebound after $\mathrm{AB} / \mathrm{PD}$ inhibition, so the LP neuron begins to fire at an earlier phase during DA. DA also phase-advances the termination of LP firing by an indirect effect through the PY neurons. DA excites the PY neurons by reducing $I_{\mathrm{A}}$ (Figure 1C), which phaseadvances their onset of firing. DA also strengthens the PY $\rightarrow$ LP synapse via the complicated mechanism shown in Figure 4. As a consequence, DA both strengthens and phase-advances the timing of PY inhibition of the LP so that the LP neuron terminates its burst at an earlier phase than under control conditions. Thus, during DA, the LP $\rightarrow$ PD inhibition is strengthened, but its offset is also phase-advanced to an earlier point in the period. This is the point when the $\mathrm{AB} / \mathrm{PD}$ neurons are at their maximal hyperpolarization after their burst, and are minimally sensitive to additional inhibition, corresponding to the null point in the phase-response curves. Thus, the phase advance of LP inhibition during DA explains the loss of LP regulation of cycle frequency during DA. Thurumalai et al. (2006) described a similar effect with the peptide red pigment-concentrating hormone (RPCH), which strengthens the LP $\rightarrow$ PD synapse but has no effect on LP regulation of cycle period. As we found with DA, during RPCH, the LP neuron fires at the null point of the phase-response curve, where the additional inhibition does not modify the timing of the next AB/PD burst. Thurumalai et al. (2006) argue that this will help stabilize the cycle frequency of the network, since any change in frequency would move the timing of the $\mathrm{LP} \rightarrow \mathrm{PD}$ inhibition away from the null point of the PRC, which would force the frequency back to its set point during RPCH.

A second example is also seen with the LP $\rightarrow$ PD synapse. Serotonin inhibits the LP neuron by a direct action (Flamm and Harris-Warrick, 1986b; Figure 6A), and also weakens the LP $\rightarrow$ PD synapse when measured in isolation (Johnson et al., 1994; Figure 6B). Thus, we would expect that serotonin would reduce the effectiveness of the LP neuron in regulating the pyloric cycle frequency. Under control conditions, removal of LP inhibition typically accelerated the pyloric rhythm (Figure 6C). However, during 5-HT application, the LP synapse's effect on cycle frequency was not reduced as expected, but was enhanced: removal of the LP neuron during 5-HT administration more strongly accelerated the pyloric rhythm than under control conditions (Figure 6C). The mechanisms driving this paradoxical result once again arise one step away in the pyloric network to alter the phasing of the LP $\rightarrow$ PD inhibition. The PY neurons normally terminate the LP burst during the pyloric rhythm. Serotonin significantly weakens the PY $\rightarrow$ LP synapse (Johnson et al., 1995; Figure 6D); this allows the LP neuron to fire for a longer phase of the cycle than under control conditions (Figure 6C), and thus 
A

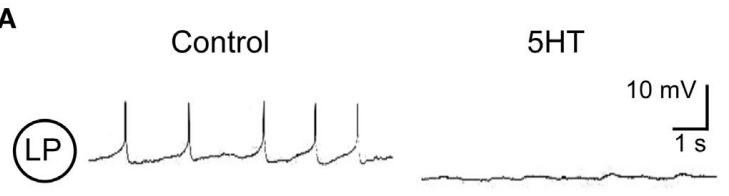

C

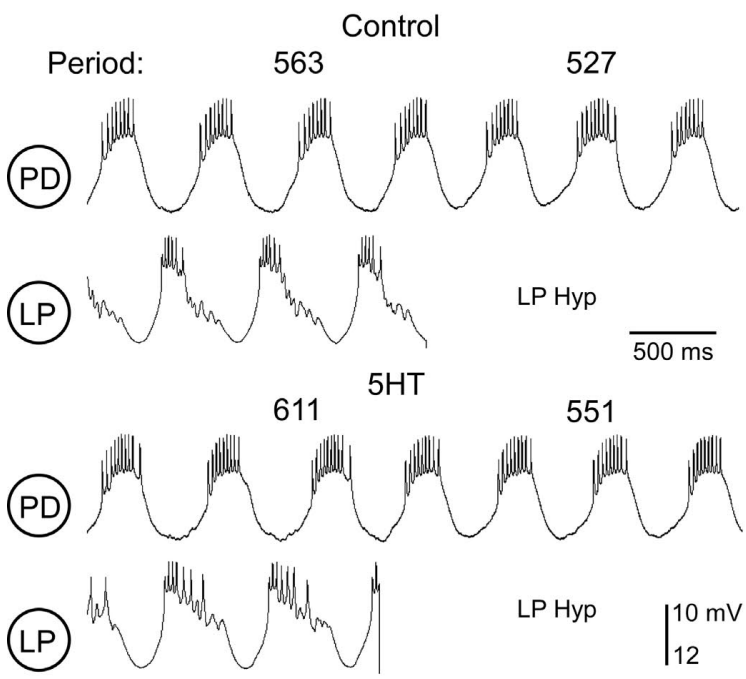

B
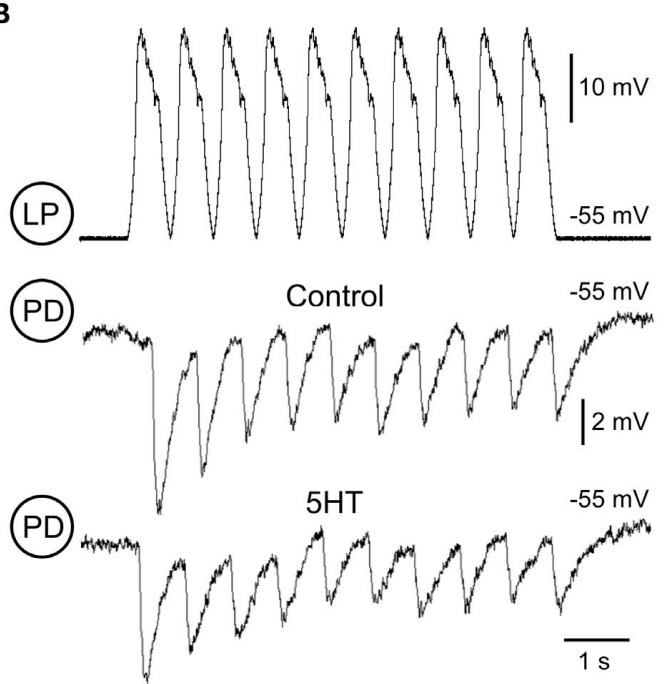

D

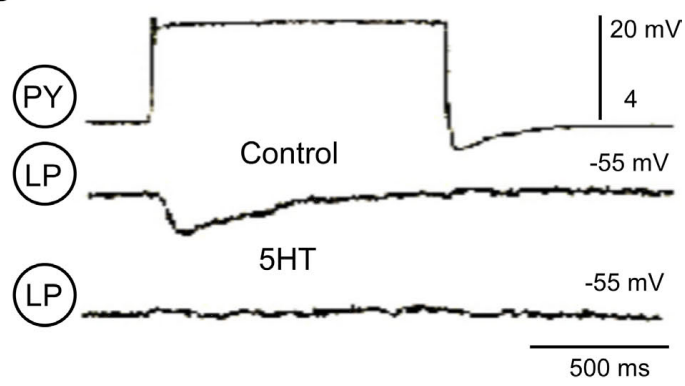

FIGURE 6 | Serotonin (5-HT; $\left.10^{-5} \mathrm{M}\right)$ modulation of LP effectiveness in the pyloric network. (A) $5-\mathrm{HT}$ inhibition of a synaptically isolated LP neuron (modified from Flamm and Harris-Warrick, 1986b). (B) Pre-synaptic LP depolarization with realistic waveform stimulation (top) and PD responses under control conditions (middle) and during application of 5-HT (bottom). (C) Effects of the LP synapse on pyloric period under control and 5-HT conditions. The top two

continue to inhibit the $\mathrm{AB} / \mathrm{PD}$ pacemaker neurons for a longer time, and at a later time on the phase-response curve. Even though the LP $\rightarrow$ PD synaptic inhibition is weaker, it lasts longer, and as a result delays the rebound of the $\mathrm{AB} / \mathrm{PD}$ neurons to the next burst. When the LP neuron is removed, the pacemaker neurons are liberated from this prolonged inhibition and show a more significant acceleration of cycle frequency than under control conditions. Thus, 5-HT enhances the LP neuron's frequency regulation despite serotonergic inhibition of the LP neuron and its synapses, due to an indirect disinhibition of the LP burst firing duration. Changing the timing of the LP inhibition in these two cases appears to be functionally more important than changing the strength of the synapse, as predicted from the modeling study of Prinz et al. (2003).

\section{FUNCTIONAL SIGNIFICANCE OF OPPOSING ACTIONS OF NEUROMODULATORS}

As can be seen in these examples, it is not rare to find a neuromodulator exerting opposing effects at a single site in a neural network. What is the functional significance of these opposing effects? We propose four possible explanations. traces show examples of PD and LP activity before and after LP hyperpolarization (LP Hyp) under control conditions. Bottom traces show the same activity and responses during 5-HT application. Measurements of the cycle period before and after LP Hyp are given above the PD traces. (D) The PY graded inhibition of LP is greatly weakened during 5-HT application (modified from Johnson et al., 1994).

First, the opposing effects could merely reflect evolutionary noise as a consequence of the biochemical cascades activated by metabotropic receptors. Activation of any second messenger pathway will modify the activities of multiple proteins in a neuron (for example, those with cAMP-regulated phosphorylation sites). Provided that the net effect of the neuromodulator is achieved, it may not matter that there are also minor opposing actions. The neuromodulatory response would be a "majority rule" of the major effects over the minor ones.

Second, opposing effects could provide for flexibility in the sign of the effect of a neuromodulator, depending on the ongoing state of the system. Any mechanism which weakens or negates one of the two opposing actions would uncover the unbalanced effect of the other action. Other neuromodulators may block one of the opposing actions. For example, serotonin activates both a $\mathrm{K}_{\mathrm{IR}}$ current and $\mathrm{Ca}_{\mathrm{V}}$ current in the Aplysia neuron $\mathrm{R} 15$, resulting in a shift from bursting to bistability as the two currents differentially stabilize the down- or up-state of the neuron (Levitan and Levitan, 1988). DA reduces serotonin's elevation of $I_{\mathrm{Ca}(\mathrm{V})}$, which could convert serotonin's net effect from plateau induction to a net inhibitory action. 
Third, the multiple effects of a neuromodulator may only be apparently opposing, while in reality acting over different concentration, voltage, kinetic, or spatial ranges. In the R15 example, serotonin's enhancement of $I_{\mathrm{KIR}}$ and $I_{\mathrm{Ca}(\mathrm{V})}$ occur over non-overlapping voltage ranges, so they do not oppose one another but rather stabilize two different states of the neuron to support bistability (Levitan and Levitan, 1988); in addition, at low concentrations of serotonin, only the inhibitory enhancement of $I_{\mathrm{KIR}}$ is observed. In the VD neuron, brief pressure application of DA shows that its opposing actions to excite and inhibit the neuron operate over different time scales, resulting in a biphasic inhibitory-excitatory response rather than some intermediate state (Harris-Warrick et al., 1998). Thus, the concentration and voltage range and the temporal dynamics of the modulatory effects must be taken into account in assessing opposing actions of neuromodulators.

Finally, a neuromodulator may indeed evoke opposing actions with the goal of providing a system of checks and balances as feedback to stabilize the new modulated state of the system. While network flexibility is essential for the production of adaptive behavior, it carries with it the threat of network instability, as an "overmodulated" network could become unstable and cease to function effectively (Grashow et al., 2009). Thus, constraints on the degree of modulation, and active mechanisms for stabilization of the modulated state may be essential to limit the system's flexibility within the behaviorally relevant parameter space. Seen

\section{REFERENCES}

Ayali, A., and Harris-Warrick, R. M. (1999). Monoamine control of the pacemaker kernel and cycle frequency in the lobster pyloric network. J. Neurosci. 19, 6712-6722.

Ayali, A., Johnson, B. R., and HarrisWarrick, R. M. (1998). Dopamine modulates graded and spike evoked synaptic inhibition independently at single synapses in the pyloric network of the lobster. J. Neurophysiol. 79, 2063-2069.

Balu, R., and Strowbridge, B. W. (2007). Opposing inward and outward conductances regulate rebound discharges in olfactory mitral cells. J. Neurophysiol. 97, 1959-1968.

Cleland, T. A., and Selverston, A. I. (1997). Dopaminergic modulation of inhibitory glutamate receptors in the lobster stomatogastric ganglion. J. Neurophysiol. 78, 3450-3452.

Endo, T., and Kiehn, O. (2008). Asymmetric operation of the locomotor central pattern generator in the neonatal mouse spinal cord. $J$. Neurophysiol. 100, 3043-3054.

Flamm, R. E., and Harris-Warrick, R. M. (1986a). Aminergic modulation in the lobster stomatogastric ganglion. I. Effects on the motor pattern and activity of neurons within the pyloric circuit. J. Neurophysiol. 55, 847-865.

Flamm, R. E., and Harris-Warrick, R. M. (1986b). Aminergic modulation in

from this perspective, the opposing actions of neuromodulators that we have described act as a system of checks and balances to protect the network from overmodulation. For example, the $\mathrm{LP} \rightarrow \mathrm{PD}$ synapse provides the only inhibitory feedback to regulate the pacemaker kernel of the pyloric network. If this synapse were to grow too strong during DA application, it could abolish the pyloric rhythm; thus, the opposing pre-synaptic enhancement and post-synaptic inhibition of this synapse by DA may act to stabilize the strengthened synapse at a level which is appropriate for the DA-modulated motor pattern. Similarly, DA excites the $\mathrm{AB}$ and $\mathrm{PY}$ neurons and increases their firing rates, which could drive enough synaptic inhibition to disrupt the motor pattern; DA's enhancement of $I_{\mathrm{K}(\mathrm{V})}$ may act to prevent the neurons from firing at a non-adaptive rate. From an engineering perspective, such a series of checks and balances is essential for any system that requires both flexibility and stability; as a consequence, the modulators' opposing effects may have been evolutionarily selected to assure appropriate behaviors. In this case, opposing neuromodulator actions should not be rare, and would be more and more necessary in larger and more complex systems to help assure stability with flexibility.

\section{ACKNOWLEDGMENTS}

Supported by NIH grant NS17323. We thank Andreas Husch and Matthew Abbinanti for useful comments on the manuscript.

Harris-Warrick, R. M., and Flamm, R. E. (1987). Multiple mechanisms of bursting in a conditional bursting neuron. J. Neurosci. 7, 2113-2128.

Harris-Warrick, R. M., and Johnson, B. R. (2002). "Cellular and molecular mechanisms of amine modulation in Crustacea," in Crustacean Experimental Systems in Neurobiology, eds K. Wiese, and J.Schmidt (Berlin: Spring Verlag), 23-44.

Harris-Warrick, R. M., Johnson, B. R., Peck, J. H., Kloppenburg, P., Ayali, A., and Skarbinski, J. (1998). Distributed effects of dopamine modulation in the crustacean pyloric network. Ann. N. Y. Acad. Sci. 860, 155-167.

Harris-Warrick, R. M., Marder, E., Selverston, A. I., and Moulins, M. (1992). Dynamic Biological Networks. The Stomatogastric Nervous System. Cambridge: MIT Press.

Johnson, B. R., and Harris-Warrick, R. M. (1997). Amine modulation of glutamate responses from pyloric motor neurons in the lobster stomatogastric ganglion. J. Neurophysiol.78, 3210-3221.

Johnson, B. R., and Hooper, S. L. (1992). "Overview of the stomatogastric nervous system," in Dynamic Biological Networks: The Stomatogastric Nervous System, eds R. M. Harris-Warrick, E. Marder, A. I. Selverston, and M. Moulins (Cambridge, MA: MIT Press), 1-30.
Johnson, B. R., Kloppenburg, P., and Harris-Warrick, R. M. (2003). Dopamine modulation of calcium currents in pyloric neurons of the lobster stomatogastric ganglion. $J$. Neurophysiol. 90, 631-643.

Johnson, B. R., Peck, J. H., and HarrisWarrick, R. M. (1994). Differential modulation of chemical and electrical components of mixed synapses in the lobster stomatogastric ganglion. J. Comp. Physiol. A 175, 233-249.

Johnson, B. R., Peck, J. H., and HarrisWarrick, R. M. (1995). Distributed amine modulation of graded chemical transmission in the pyloric network of the lobster stomatogastric ganglion. J. Neurophysiol. 74, 437-452.

King, D. G. (1976). Organization of crustacean neuropil. II. Distribution of synaptic contacts on identified motor neurons in lobster stomatogastric ganglion. J. Neurocytol. 5, 239-266.

Kloppenburg, P., Levini, R. M., and HarrisWarrick, R. M. (2008). Dopamine modulates two potassium currents and inhibits the intrinsic firing properties of an identified motor neuron in a central pattern generator network. $J$. Neurophysiol. 81, 29-38.

Kloppenburg, P., Zipfel, W. R., Webb, W. W., and Harris-Warrick, R. M. (2007). Heterogeneous effects of dopamine on highly localized, voltage-induced $\mathrm{Ca}^{2+}$ accumulation in identified motoneurons. J. Neurophysiol. 98, 2910-2917. 
Levitan, E. S., Kramer, R. H., and Levitan, I. B. (1987). Augmentation of bursting pacemaker activity by egg-laying hormone in Aplysia neuron R15 is mediated by a cyclic AMP-dependent increase in $\mathrm{Ca}^{2+}$ and $\mathrm{K}^{+}$currents. Proc. Natl. Acad. Sci. U.S.A. 84, 6307-6311.

Levitan, E. S., and Levitan, I. B. (1988). Serotonin acting via cyclic AMP enhances both the hyperpolarizing and depolarizing phases of bursting pacemaker activity in the Aplysia neuron R15. J. Neurosci. 8, 1152-1161.

Lu, J., Dalton, J. F. IV, Stokes, D. R., and Calabrese, R. L. (1997). Functional role of $\mathrm{Ca}^{2+}$ currents in graded and spike-mediated synaptic transmission between leech heart interneurons. J. Neurophysiol. 77, 1779-1794.

MacLean, J. N., Zhang, Y., Johnson, B. R., and Harris-Warrick, R. M. (2003). Activity-independent homeostasis in rhythmically active neurons. Neuron 37, 109-120.

Marder, E., and Bucher, D. (2007). Understanding circuit dynamics using the stomatogastric nervous system of lobsters and crabs. Annu. Rev. Physiol. 69, 291-316.

Peck, J. H., Gaier, E., Stevens, E., Repicky, S., and Harris-Warrick, R. M. (2006). Amine modulation of Ih in a small neural network. J. Neurophysiol. 96, 2931-2940.

Peck, J. H., Nakanishi, S. T., Yaple, R., and Harris-Warrick, R. M. (2001). Amine modulation of the transient potassium current in identified cells of the lobster stomatogastric ganglion. J. Neurophysiol. 86, 2957-2965.

Power, J. M., and Sah, P. (2008). Competition between calciumactivated $\mathrm{K}^{+}$channels determines cholinergic action on firing properties of basolateral amygdala pro- jection neurons. J. Neurosci. 28, 3209-3220.

Prinz,A.A., Thirumalai, V., and Marder, E. (2003). The functional consequences of changes in the strength and duration of synaptic inputs to oscillatory neurons. J. Neurosci. 23, 943-954.

Schulz, D. J., Goaillard, J. M., and Marder, E. (2007). Quantitative expression profiling of identified neurons reveals cellspecific constraints on highly variable levels of gene expression. Proc. Natl. Acad. Sci. U.S.A. 104, 13187-13191.

Thurumalai, V., Prinz, A. A., Johnson, C. D., and Marder, E. (2006). Red pigment concentrating hormone strongly enhances the strength of the feedback to the pyloric rhythm oscillator but has little effect on pyloric rhythm period. J. Neurophysiol. 95, 1762-1770.

Turrigiano, G. G. (2008). The self-tuning neuron: synaptic scaling of excitatory synapses. Cell 135, 422-435.
Conflict of Interest Statement: The authors declare that the research was conducted in the absence of any commercial or financial relationships that could be construed as a potential conflict of interest.

Received: 14 April 2010; paper pending published: 11 June 2010; accepted: 02 July 2010; published online: 21 July 2010.

Citation: Harris-Warrick RM and Johnson BR (2010) Checks and balances in neuromodulation. Front. Behav. Neurosci. 4:47. doi: 10.3389/fnbeh.2010.00047

Copyright (c) 2010 Harris-Warrick and Johnson. This is an open-access article subject to an exclusive license agreement between the authors and the Frontiers Research Foundation, which permits unrestricted use, distribution, and reproduction in any medium, provided the original authors and source are credited. 